\title{
McCarraher, Eugene: The Enchantments of Mammon. How Capitalism Became the Religion of Modernity, 816 S., Harvard UP, Cambridge, MA/London 2019.
}

\author{
Jürgen Osterhammel
}

Online publiziert: 8 . Oktober 2020

(C) Der/die Autor(en) 2020

Dass dieses Buch in einem führenden Universitätsverlag erschienen ist, hat seine Berechtigung und führt dennoch in die Irre. Denn während es auf der einen Seite eine grundgelehrte Studie zur Ideen- und Kulturgeschichte ist, handelt es sich zugleich um eine Anklageschrift gegen eine gesamte Zivilisation, unsere eigene Zivilisation des Raubbaus, der Technikvergötzung und der Gier nach Konsumerlebnissen und Reichtumsmaximierung. Die Anklage mündet auf den letzten Seiten in eine grandiose Bußpredigt angesichts drohender ökologischer und ökonomischer Katastrophen, in einen Ruf zur Abkehr von ,corporate plutocracy“ (S. 663) und zur Umkehr vor „our descent into the hell of property, rank and dominion“ (S. 674). Derlei richtet sich an die breitestmögliche politische Öffentlichkeit, nicht nur an die typischen Leser von Harvard University Press.

Was in der resümierenden Zuspitzung eines Schlusskapitels schrill und dramatisierend klingt, ist eine wohlüberlegte Antwort auf eine Krise, die Eugene McCarraher noch nicht kennen konnte, als er in 20-jähriger Arbeit sein Buch recherchierte und schrieb. Die Pandemie von 2020 hat jene extremen Ungleichheiten innerhalb der US-amerikanischen Gesellschaft und jene enorme Verletzlichkeit der Schwächeren in dieser Gesellschaft in ein helles Licht gerückt, die McCarraher so wortgewaltig geißelt. Er steht selbst in der Tradition der Kapitalismuskritik, die er mit großer Ausführlichkeit beschreibt und dokumentiert. Genauer gesagt: Diese Tradition ,gibt‘ es nicht, er muss sie erst (re)konstruieren.

Selbstverständlich ist Karl Marx dabei eine zentrale Figur, der Marx der Entfremdungskritik, der „Grundrisse“ und der Theorie des Warenfetischismus aus dem „Kapital“. Marx erkannte auch wie niemand vor ihm ,the metaphysics of money“ (S. 59). Doch war er mit seiner Fixierung auf Produktion und Produktivität zu

J. Osterhammel $(\bowtie)$

Albert-Ludwigs-Universität Freiburg im Breisgau, Freiburg i. Br., Deutschland

E-Mail: juergen.osterhammel@ frias.uni-freiburg.de 
stark an die Fortschrittsidee gebunden, und er erwartete irrtümlich - so liest ihn McCarraher - das Verschwinden aller Illusionen und ideologischen Vernebelungen mit der fortschreitenden Rationalisierung der Kapitallogik und ihrem schließlichen Umschlag in den Sozialismus.

Eben dies geschah aber nicht. Der Kapitalismus brutalisierte und barbarisierte zwar die Welt (McCarraher ist dabei nicht so blind, die wohlstandssteigernden Wirkungen der kapitalistischen Produktionsweise zu leugnen), ,entzauberte‘ sie jedoch keineswegs. Im Gegenteil, er beruht bis zum heutigen Tage auf Fremdtäuschung und Selbsttäuschung, auf der unentwegten Produktion von Imaginationen, etwa durch Werbung, Mythenbildung um die Institution des Großkonzerns, Fordismus oder die Pseudo-Philosophien angeblich grenzenlos wohltätiger, unumkehrbarer Globalisierung und Finanzialisierung. Zu all diesen Themen hat der Autor bemerkenswertes Material zusammengetragen und es mit Scharfsinn und einer Spur Sarkasmus analysiert. Das Buch ist eine überaus ergiebige Vorstellungsgeschichte der kapitalistischen Konsumgesellschaft in den USA und damit im Prinzip überall in der westlichen Welt. Die gelungensten Kapitel behandeln die Zeit seit dem Zweiten Weltkrieg.

Trotz der Marx'schen Analyse des ,Warenfetischismus', die übrigens im späteren sozialistischen Denken keine große Rolle mehr spielte, war es in McCarrahers Sicht nicht die systemkritische Linke, die das kapitalistische imaginaire, vor allem die Sakralisierung des Geldes, am klarsten durchschaute und ihm Gegenentwürfe entgegenstellte, sondern das, was er „Romantik“ nennt. Das ist keine kohärente Denkrichtung oder Schule und geht auch weit über die ästhetische und politische Romantik des frühen 19. Jahrhunderts hinaus. Die „Romantiker“ in diesem Buch sind eine lose Reihe von Denkern, denen gemeinsam ist, dass sie, mit einer etwas schlichten Formel von Ernst Fromm, das ,Sein“ über das ,Haben“ stellen. Auch denken sie eher lokal als global, eher konkret als abstrakt, eher qualitativ als quantitativ; sie betrachten die Natur und die Welt materieller Gegenstände mit Demut und Respekt und nicht als Objekt von Rücksichtslosigkeit und Ausbeutung; das Handwerk wahrt seine Würde gegenüber der Fabrikproduktion. Dem ,Markt“ mit seinen angeblichen Gesetzmäßigkeiten vertrauen sie weniger, als dies für die gesamte Tradition der Politischen Ökonomie charakteristisch ist; Gemeinschaftsdenken und eine moral economy gegenseitiger Verpflichtung werden umso höher geschätzt, manchmal im Rückblick auf die Vormoderne nostalgisch verklärt. Die Galerie dieser Dissidenten reicht von Gerard Winstanley vom radikalen Rand der Englischen Revolution des 17. Jahrhunderts über Thomas Carlyle, John Ruskin und William Morris im England des 19. Jahrhunderts bis zu dem amerikanischen Universalgelehrten Lewis Mumford, dem Theoretiker der „Gegenkultur“" Theodore Roszak oder auch Papst Franziskus und seiner Enzyklika „Laudato si”“ von 2015.

„Enchantment“ bedeutet in diesem Buch nicht nur die fortdauernde und meist manipulativ erzeugte Ablenkung der Menschen vom Wesentlichen, sondern ebenso etwas ganz anderes, das nicht deutlich genug vom ersten Wortsinn unterschieden wird: Anders als Max Weber einflussreich meinte (ohne sich ganz sicher zu sein), hat die kapitalistische Moderne die ,Verzauberung ' durch das Religiöse keineswegs endgültig wegrationalisiert. Wir leben, sagt McCarraher, weiterhin in der Präsenz Gottes und werden ihn klarer erkennen, wenn wir uns vom Unheil unserer gegenwärtigen Lebensführung befreit haben. Die theologische Argumentationsebene 
gehört integral zu diesem mehrschichtigen Werk. Auch wer „religiös unmusikalisch“ (Max Weber) ist, sollte sich von der Auseinandersetzung mit ihm nicht abschrecken lassen. Es zählt neben Thomas Pikettys neuem Buch, das ebenfalls zum Teil ideengeschichtlich vorgeht („Capital et idéologie“, 2019, dt. 2020), zu den Leittexten gegenwärtiger Kapitalismuskritik.

Funding Open Access funding enabled and organized by Projekt DEAL.

Open Access Dieser Artikel wird unter der Creative Commons Namensnennung 4.0 International Lizenz veröffentlicht, welche die Nutzung, Vervielfältigung, Bearbeitung, Verbreitung und Wiedergabe in jeglichem Medium und Format erlaubt, sofern Sie den/die ursprünglichen Autor(en) und die Quelle ordnungsgemäß nennen, einen Link zur Creative Commons Lizenz beifügen und angeben, ob Änderungen vorgenommen wurden.

Die in diesem Artikel enthaltenen Bilder und sonstiges Drittmaterial unterliegen ebenfalls der genannten Creative Commons Lizenz, sofern sich aus der Abbildungslegende nichts anderes ergibt. Sofern das betreffende Material nicht unter der genannten Creative Commons Lizenz steht und die betreffende Handlung nicht nach gesetzlichen Vorschriften erlaubt ist, ist für die oben aufgeführten Weiterverwendungen des Materials die Einwilligung des jeweiligen Rechteinhabers einzuholen.

Weitere Details zur Lizenz entnehmen Sie bitte der Lizenzinformation auf http://creativecommons.org/ licenses/by/4.0/deed.de.

\title{
Robichaud, Andrew A.: Animal City. The Domestication of America, 352 S., Harvard UP, Cambridge, MA/London 2019.
}

\author{
Andreas Hübner \\ Online publiziert: 22. September 2020 \\ (C) Der/die Autor(en) 2020
}

Den Blick auf die Flut an Arbeiten gerichtet, die sich dem Themenfeld der HumanAnimal Studies widmeten, sprach die Historikerin Harriet Ritvo im Jahr 2007 von einem animal turn in den Geistes- und Sozialwissenschaften. Über ein Jahrzehnt später hält die Konjunktur der Human-Animal Studies an. In ihrem Sog haben sich auch die historischen Tierforschungen maßgeblich konturiert, professionalisiert und institutionalisiert. Zumeist den Prämissen sozial- und kulturgeschichtlicher Ansätze

\footnotetext{
A. Hübner $(\bowtie)$

Leuphana Universität Lüneburg, Lüneburg, Deutschland

E-Mail: andreas.huebner@leuphana.de
} 